To illustrate, consider the area of a sphere: Suppose we start with a cube whose edges are ro centimeters. By continual removal of vertices we approach a sphere whose diameter is Io centimeters. Remove the 8 vertices of the cube by means of planes tangent to the sphere. We then obtain a I4-sided figure, the area of whose surface is less than 600 centimeters. Denote this area by $s_{1}$. Again remove all the vertices of this figure by means of planes tangent to the sphere thus obtaining a new figure with surface area $s_{2}$. Proceeding in this manner we obtain a decreasing sequenee $s_{1}, s_{2}, s_{3}$. which is bounded and hence defines a definite number. This number we define as the area of the sphere.

A proof that this is the area of the surface is as impossible here as in the case of the circle and for exactly the same reasons. It is then a matter of proof that the area so defined is equal to $4 \pi r^{2}$, etc.

\title{
CULTURE COURSE. (III.)
}

By T. M. BLAKsLEe.

Central City, Lowa.

(Continued from the December Number, p. 755.)

Part III, Sec. I. Introduction.

(a). Simple Equation. Nothing new needed.

(b). Linear Equations with two unknowns.

$a x+b y=0(1) a^{\prime} a x+a^{\prime} b y=a^{\prime} c$
$a^{\prime} x+b^{\prime} y=c^{\prime}(2) a a^{\prime} x+a b^{\prime} y=a c^{\prime} y=\frac{a c^{\prime}-a^{\prime} c}{a b^{\prime}-a^{\prime} b}$

The numerator is the algebraic sum of the products of the

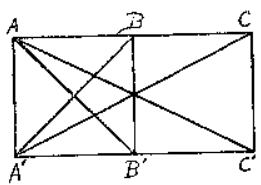
diagonal elements in the entire rectangle The denominator is the like sum for the left partial rectangle. The sign of the product is opposite to that of the slant of the diagonal.

Solve mentally:
(I). $2 \mathrm{x}+2 \mathrm{y}=\mathrm{I} 7$
(2). $\quad 2 x+y=9$
$4 \mathrm{x}+\mathrm{y}=\mathrm{I} 6$
$5 x+3 y=25$
(3). $\frac{10}{x}+\frac{9}{y}=8$
(4). $\frac{a}{x}+\frac{b}{y}=c$
$\frac{8}{\mathrm{x}}+\frac{\mathrm{I} 5}{\mathrm{y}}=9$
$\frac{b}{x}+\frac{a}{y}=c$

(5) $2 m p+n q=k, 3 m p-5 n q=r$;

Solve, first for $p$ and $q$, then for $m$ and $n$. 
(c). Linear Equations is any number of unknowns.

$\mathrm{A}_{\mathrm{I}} \mathrm{x}_{1}+\mathrm{B}_{1} \mathrm{x}_{2}+\mathrm{C}_{1} \mathrm{x}_{2}+\ldots \ldots \quad \mathrm{L}_{1} \mathrm{x}_{\mathrm{n}}=\mathrm{M}_{1}$

$\mathrm{A}_{2} \mathrm{x}_{1}+\mathrm{B}_{2} \mathrm{x}_{2}+\mathrm{C}_{2} \mathrm{x}_{3}+\ldots \ldots \quad \mathrm{L}_{2} \mathrm{x}_{\mathrm{n}}=\mathrm{M}_{\mathrm{y}}$

$A_{n} x_{1}+B_{n} x_{2}+C_{n} x_{3}+\ldots . \quad L_{2} x_{n}=M_{n}$ (n)

By (I) $x_{1}=\frac{M_{1}}{A_{1}}-\frac{B_{1}}{A_{1}} x_{2}-\frac{C_{1}}{A_{1}} x_{3} \ldots \ldots \frac{L_{1}}{A_{1}} x_{n}$. Hence

$\left(A_{1} B_{2}-A_{2} B_{1}\right) x_{2}+\left(A_{1} C_{2}-A_{2} C_{1}\right) x_{3}+\ldots \ldots=A_{1} M_{2}-A_{2} M_{2}$

Similarly for $(3)^{1} \ldots \ldots\left(\mathbf{n}^{1}\right)$.

Definition: $\left|\begin{array}{ll}a_{1}, & b_{1} \\ a_{2}, & b_{2}\end{array}\right|=a_{1} b_{2}-a_{2} b_{1}$.

Solve :
(I) $\left\{\begin{aligned} x+2 y+z & =12 \\ 2 x-3 y+4 z & =11 \\ x+5 y+2 z & =3\end{aligned}\right.$
(2) $\left\{\begin{array}{c}-7 y+2 z=-13 \\ y-3 z=-9 \\ z=4\end{array}\right.$
(3) $\left\{\begin{aligned} 2 x+3 y+z & =11 ; \\ 5 x+y+z & =13 \\ x+2 y+3 z & =14\end{aligned}\right.$
(4) $\left\{\begin{array}{l}a_{1} x+b_{1} y+c_{1} z=d_{1} \\ a_{2} x+b_{2} y+c_{2} z=d_{2} \\ a_{3} x+b_{3} x+c_{3} z=d_{3}\end{array}\right.$.

APPLICATIONS.

Problem--To find the condition that three linear equations containing but two unknowns shall be consistent.

$$
\text { Given } \begin{array}{r}
a_{1} x+b_{1} y+c_{1}=0 \\
a_{2} x+b_{2} y+c_{2}=0 \\
a_{3} x+b_{3} y=c_{3}=0 \\
a_{1} x+b_{1} y=-c_{1}(I)^{\prime} \\
a_{2} x+b_{2} y=-c_{2}\left(2^{\prime}\right) \\
\left|\begin{array}{ll}
a_{1}, b_{1}, c_{1} \\
a_{2}, b_{2}, c_{2} \\
a_{3}, b_{3}, c_{3}
\end{array}\right|=0 .
\end{array}
$$

Test the following:

$$
\begin{array}{ll}
2 x+3 y+1=0 & 2 x+3 y+4=0 \\
3 x+4 y+1=0 & 3 x+2 y+4=0 \\
4 x+5 y+1=0 & 4 x+3 y+2=0 .
\end{array}
$$

(d) Second Degree, One Unknown (Quadratics).

Type Equation: $\mathrm{x}^{2}+2 \mathrm{px}=\mathrm{q}$

$$
\begin{gathered}
x_{1}^{2}+2 p x+p^{2}=q+p^{2} \\
-p+\sqrt{q+p^{2},} x_{2}=-p-\sqrt{q+p^{2}} \\
x_{1}+x_{2}=2 p, x_{1} x_{2}=-q
\end{gathered}
$$

$(x-a)(x-b)=0$ has $a$ and $b$ as roots, i.e., these numbers, (when substituted for $x$ ), satisfy the equation. 
Form io equations with special values for $\mathrm{a}$ and $\mathrm{b}$ and test by solving.

Second Type: $a x^{2}+b x+c=0$ gives

$$
x=\frac{-b+\sqrt{b^{2}-4 a c}}{2 a}
$$

The roots are (I) Real and Unequal, (2) Real and Equal, (3) Imaginary, (i.e., transverse) according as

$$
\mathrm{b}^{2} \stackrel{>}{=} 4 \mathrm{ac} .
$$

Polynomials in the Determinant Form.

$\left|\begin{array}{ll}a_{1}, & b_{1} \\ a_{2} & b_{2}\end{array}\right|=a_{1} b_{2}-a_{2} b_{1}$ is of the second order.

$\left|a_{1}, b_{1}, c_{1}\right|$

$a_{2}, b_{2}, c_{2}=a_{1} b_{2} c_{2}+a_{z} b_{5} c_{1}+a_{3} b_{1} c_{2}$

$a_{3}, b_{8}, c_{3} \mid-a_{1} b_{3} c_{2}-a_{2} b_{1} c_{3}-a_{3} b_{2} c_{1}$ of third order.

The science of these abbreviated forms is the Theory of Determinants.

All we need to observe here is that the first member denotes the algebraic sum of the products of three elements, one, and but one, being taken from each row and each column. The sign of a product is contrary to the slant of the ray of two of its elements.

$$
\begin{aligned}
& \left|\begin{array}{lll}
\mathrm{a}_{1}, & \mathrm{~b}_{2}, & \mathrm{c}_{2} \\
\mathrm{a}_{2}, & \mathrm{~b}_{2}, & \mathrm{c}_{2} \\
\mathrm{a}_{3}, & \mathrm{~b}_{3}, & c_{3}
\end{array}\right| \quad \text { IHI. }\left|\begin{array}{lll}
3, & 5,7 \\
4, & 2, & \mathrm{I} \\
\mathrm{I}, & \text { o, } & 7
\end{array}\right|=43 \\
& \begin{array}{r|c}
+ & - \\
42 & 14 \\
168 & 19 \\
5 & 140 \\
\hline 215- & 172 \cong 43
\end{array}
\end{aligned}
$$

(To be continued.)

\section{A PECULIAR ACTION OF THE RADIOMETER.}

$$
\text { By C. F. Adams, }
$$

Central High School, Detroit, Mich.

While arranging apparatus at one time to show selective absorption I happened to place a radiometer at the focus of light coming from the lantern so that the light was incident on the bright side of the vanes only. I was surprised to see it revolve in a direction opposite to the usual direction. Recently I repeated the experiment arranging the apparatus as shown in the figure in which $C$ represents the condenser of a lantern, $L$ an arc light, and $\mathrm{R}$ the radiometer. Two boards about $2 \mathrm{~cm}$ thick, were placed at $\mathrm{r} r$ with a space between them of about $\mathrm{I} \mathrm{cm}$. I had 